\title{
Immunohistochemical Interaction on Antisera to HCG and its Subunits with Chorionic Tissue of Early Gestation
}

\author{
Makoto HOShina, Yoshiniko ASHITAKE and Shimpei TOJO \\ Department of Obstetrics and Gynecology, Kobe University \\ School of Medicine, Kobe 950, Japan
}

\begin{abstract}
Synopsis
Immunohistochemical localization of hCG and its subunits in chorionic tissue of early gestation was carried out.

Antibodies to purified hCG and its subunits were obtained by using these agents for immunization according to the small doses method. The antibody titers and specificities were examined by $\mathrm{B} / \mathrm{T}$ and standard curves in homologous radioimmunoassay system.

The tissue preparations were stained both by a direct and by an indirect method utilizing these antisera and observing the specimens under a fluorescent microscope.

The results were as follows. 1) With the anti-hCG staining, fluorescence was observed in the syncytiotrophoblasts as reported previously while the cytotrophoblast were stained slightly.

2) with the anti-hCG- $\beta$ staining, the fluorescence was almost identical with that of hCG and showed a more distinct pattern.

3) with the anti-hCG- $\alpha$ staining, the fluorescence was found both in the syncytioand cytotrophoblasts concurrently. Fluorescence of the latter cells was recognized as due to free $\alpha$-subunit because cytotrophoblast was scarcely stained with anti-hCG and anti-hCG- $\beta$.
\end{abstract}

Although it is well known that hCG is localized to the syncytiotrophoblast of normal immature placenta, production site of hCG that is supposed to be the syncytiotrophoblast, has not yet been established.

Recently, hCG was separated into two subunits which have dissimilar antigenecity, nevertheless little work has been done to study the localization of its subunits. The histochemical demonstration of these subunits is of value to establish the site and processing of hCG. Furthermore, in parallel with the development of RIA for hCG and its subunit, it has been revealed that hCG exists in blood of patient with non-gestational malignant neoplasia and in some

Received June 14, 1978. cases of neoplasia a disproportionate amount of one subunit is produced.

Based on those findings, not only hCG but also its subunits should be examined to detect ectopic production of the peptides by neoplasia. The production of $\mathrm{hCG}$ and its subunit by malignant neoplasia may be a possible parameter of cell dedifferentiation or be a key to solve the problem of malignant transformation.

The final purpose of seriate communications is to solve the process of malignant transformation of chorionic villi.

\section{Materials and Methods}

Specimens of normal human immature placenta which contain high tissue concentration of hCG and 
its subunits and show clear pattern of recognizable syncytio- and cytotrophoblasts were studied. Chorionic villi obtained at the time of therapeutic or legal abortion were fixed in $10 \%$ buffered formalin, processed through alchol and xylol dehydration and embedded in paraffin.

As described previously, the antigen for immunization was highly purified hCG and its subunits which had identical immunochemical properties as the reference subunits obtained from Dr. Canfield (Ashitaka, 1974). Immunization procedure was performed according to the small doses method described by Vaitukaitis and associates. Namely, 50 $\mu \mathrm{g}$ of purified antigen was administered to male white rabbit (Nihon Kurea Co., Japan) intradermally at 30 to 50 sites of its back with complete Fruend's adjuvant and $2.5 \mathrm{mg}$ of tubercle bacilli (dried and killed) obtained from Toneyama National Sanatorium. Simultaneously, at another 20 to 30 sites $0.5 \mathrm{mg}$ crude Bordetella pertussis vaccine (a gift from E. Lilly Corp., Indiana, U.S.A.) was injected. Antibody titers began to increase about 45 days after the injection, and were examined by $\mathrm{B} / \mathrm{T}$ and standard curves in homologous radioimmunoassay systems as reported previously.

Immunofluorescent staining was performed both by the direct and indirect methods. Fig. 1 shows indirect staining procedure. FITC conjugated anti rabbit IgG (MBL corp., Japan, $\mathrm{F} / \mathrm{P}=1.30$ ) was used as the second labeled antibody. The procedure for the conjugation of specific antisera used in the direct method is presented in Fig. 2. Conjugata were fractionated on DEAE-Sephadex column and only fraction $1(\mathrm{~F} / \mathrm{P}=0.91-1.05)$ was used for staining. The preparations were observed for fluorescence with Zeiss standard RA fluorescent microscope and Zeiss epicondenser type fluorescent microscope.

The specificity of resulting antisera was examined by homologous radioimmunoassay systems. Fig. 3 shows that one antiserum had 100 folds or more specificity against another two antigens. FITC conjugated anti rabbit IgG (Second antiserum) was examined by immunoelectrophoresis (Fig. 4) and in a microouchterlony diffusion plate (Fig. 5). Fig. 5 shows that the antiserum reacts only with rabbit IgG and not with human serum, commercial hCG or placental homogenate.

Routine immunofluorescent control were run simultaneously as follows. 1) instead of the antisera, normal rabbit serum or PBS were used. 2) instead of the antisera, those absorbed with excess amounts of these antigens were used. 3) the process of using these antisera was omitted. 4) preparation was treated with non-labeled specific antiserum prior to the staining with the same labeled antiserum (for the direct method). 5) direct staining with aggregated FITC conjugated anti human IgG of rabbit was performed to avoid Fc-receptor. and 6) instead of human placental villi, human stomach, skin and uterus were used as specimens. With all of the control preparations, apple green fluorescence was not observed.

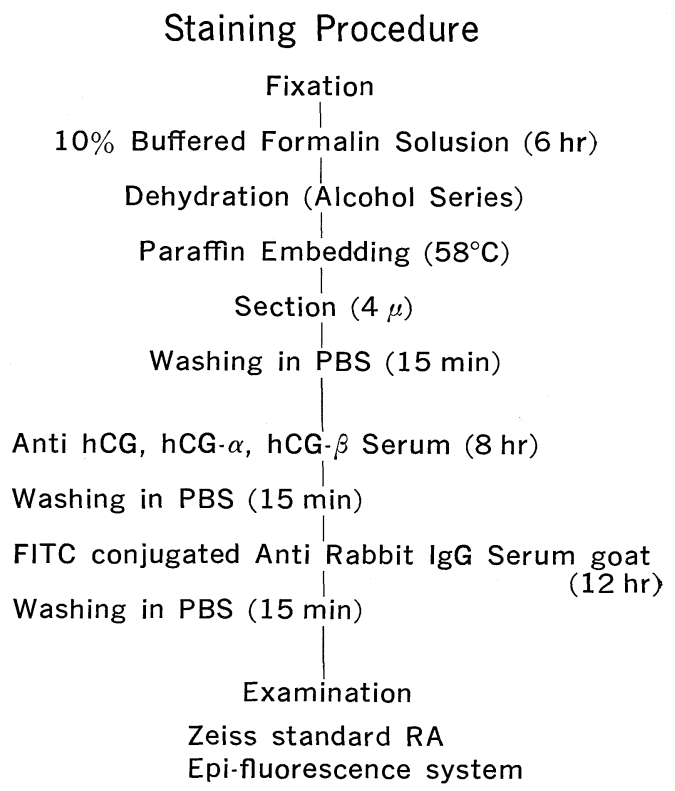

Fig. 1.

\section{Conjugation Procedure}

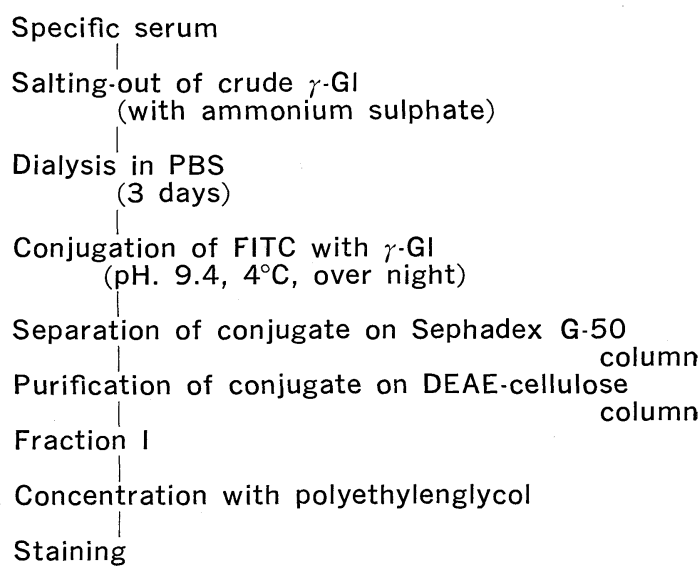

Fig. 2. 


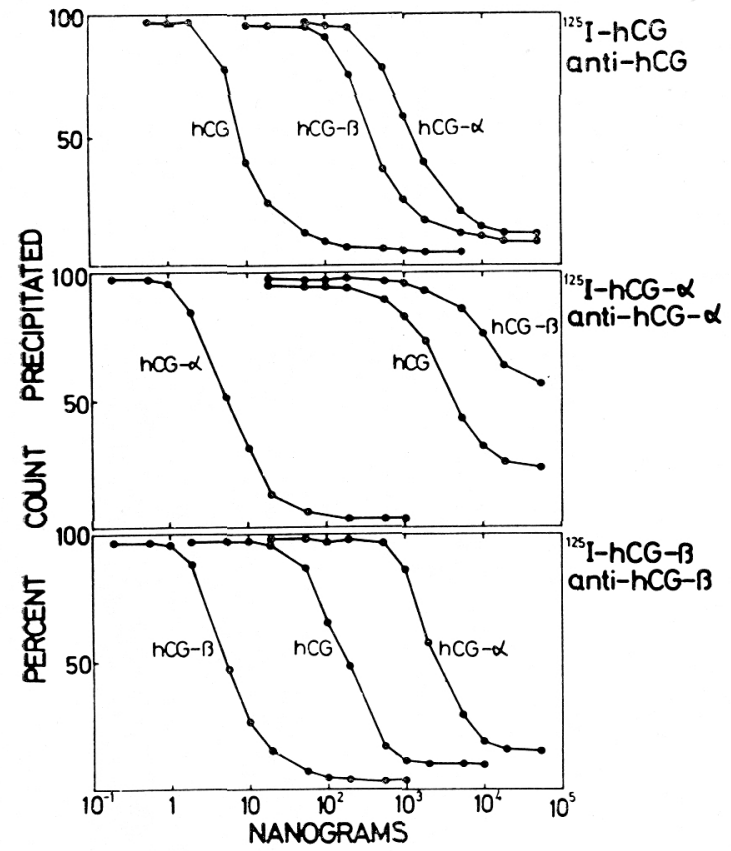

Fig. 3.

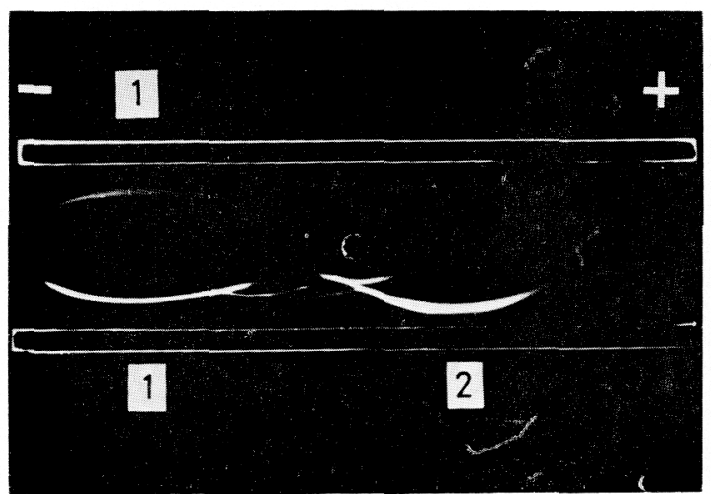

Fig. 4.

well: rabbit serum

upper trough: anti rabbit IgG (goat, FITC conj.) lower trough: anti rabbit serum 1: $\gamma$-globulin 2: albumin running conditions: veronal buffer $\mathrm{pH} 8.6$, ionic strength 0.06 $2.5 \mathrm{~mA} / \mathrm{cm}, 120 \mathrm{~min}$.

\section{Results}

The fluorescence stained with anti-hCG was observed homogenously in the cytoplasm of syncytiotrophoblast forming the outer layer of trophoblast. The syncytial bud and the trophoblast which became syncytium in the marginal area of cell column had the same fluorescence. A few number of cytotrophoblasts were also slightly stained. On the other hand, interstitial cell of chorionic villi showed no fluorescence (Fig. 6, Fig. 7). The intensity of the fluorescence in cytotrophoblast was not diminished by treatment with anti $h \mathrm{CG}-\alpha$ prior to anti $\mathrm{hCG}$ staining, as well as that in syncytiotrophoblast.

The fluorescence stained with antihCG- $\beta$ showed almost the same pattern as that stained with anti-hCG. Syncytialtrophoblast, syncytial bud and syncytiumized cells in cell column were stained and showed a more distinct border than that stained with anti hCG (Fig. 8, Fig. 9).

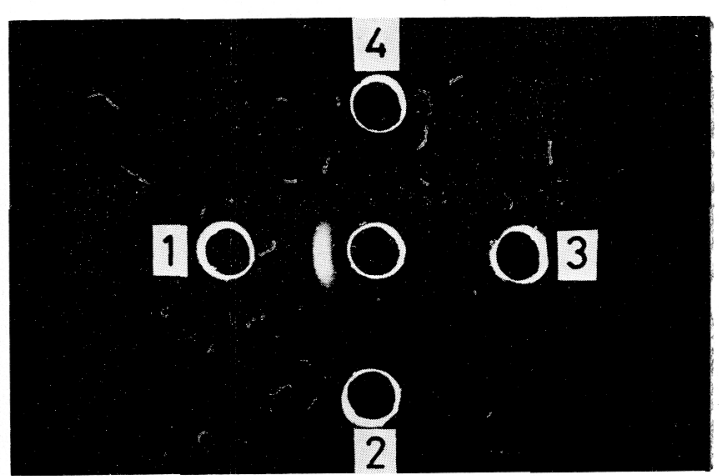

Fig. 5.

center well: anti rabbit IgG (goat, FITC conj.) 1: rabbit serum

2: commercial $\mathrm{hCG}$

3: placental homogenate

4: human serum 


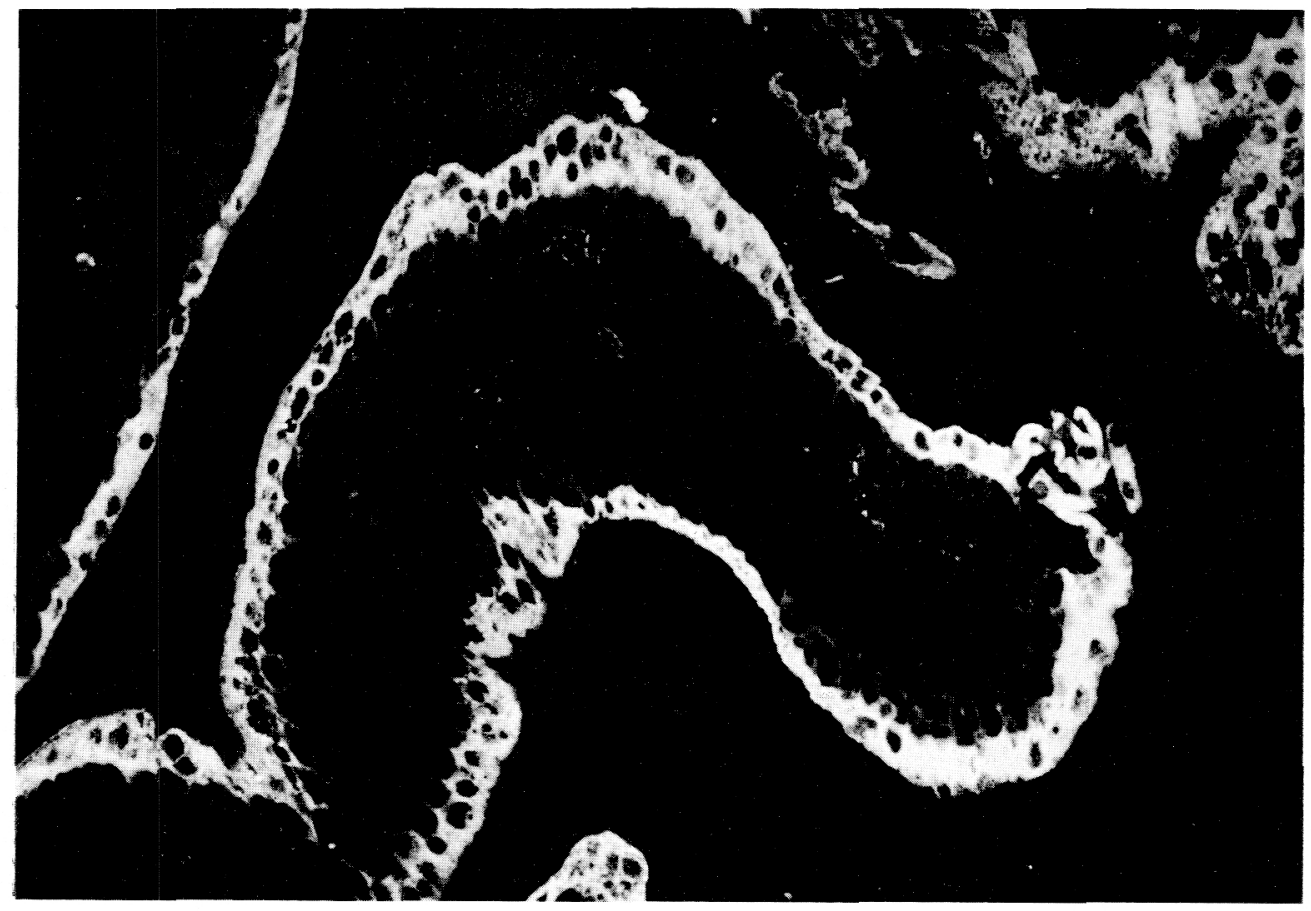

Fig. 6. Staining with anti-hCG, $\times 160$

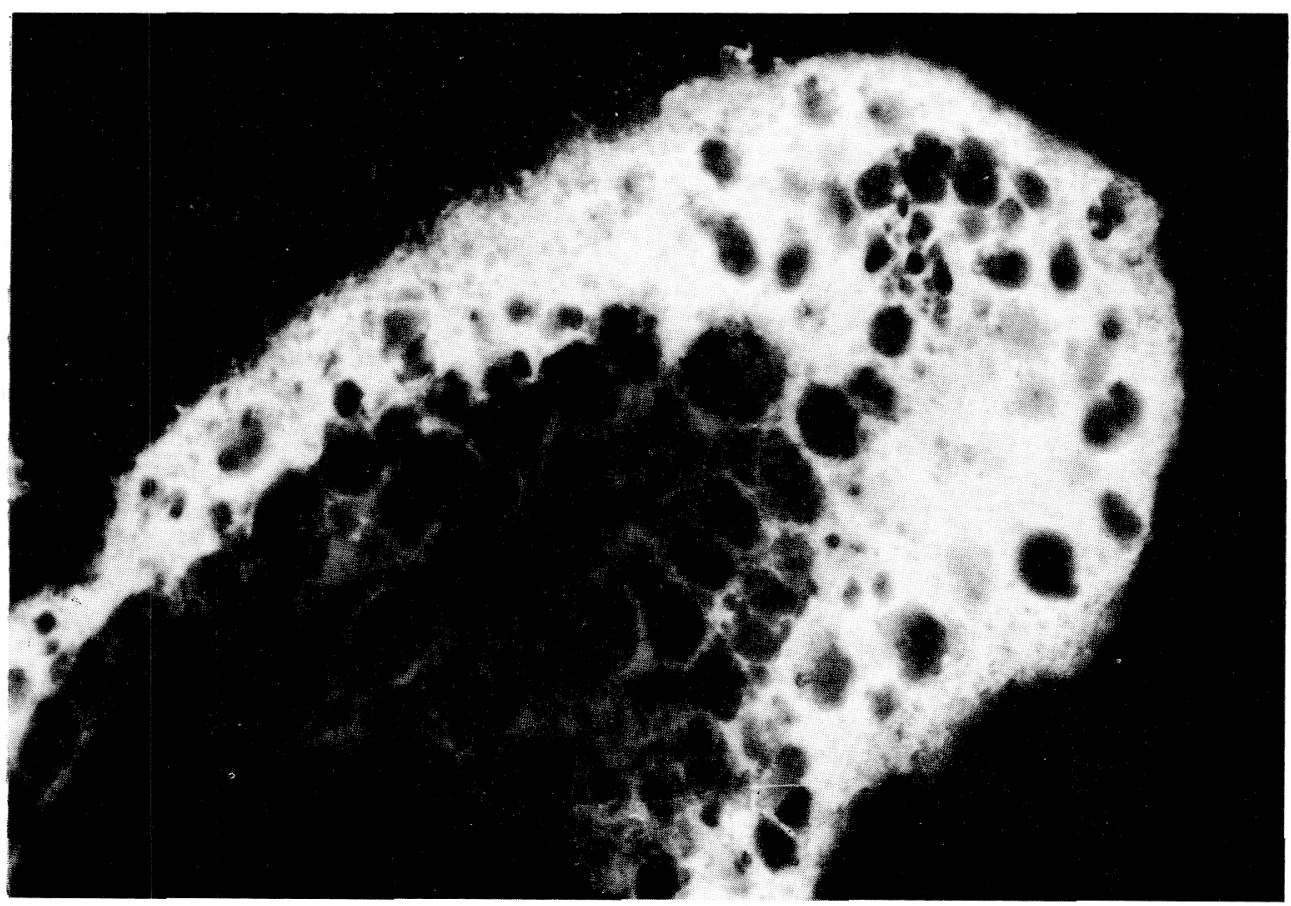

Fig. 7. Staining with anti-hCG, $\times 400$ 


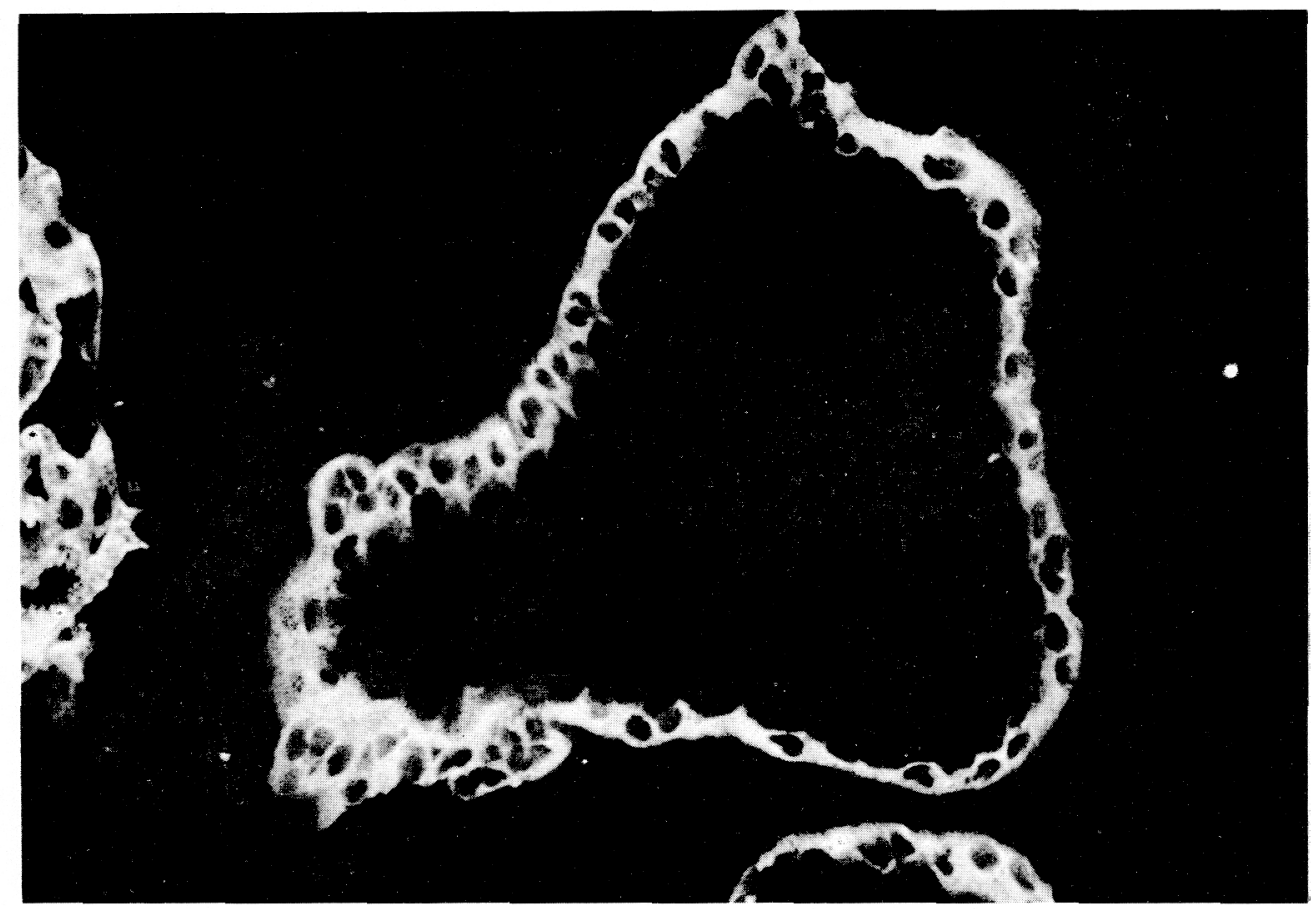

Fig. 8. Staining with anti-hCG- $\beta, \times 160$

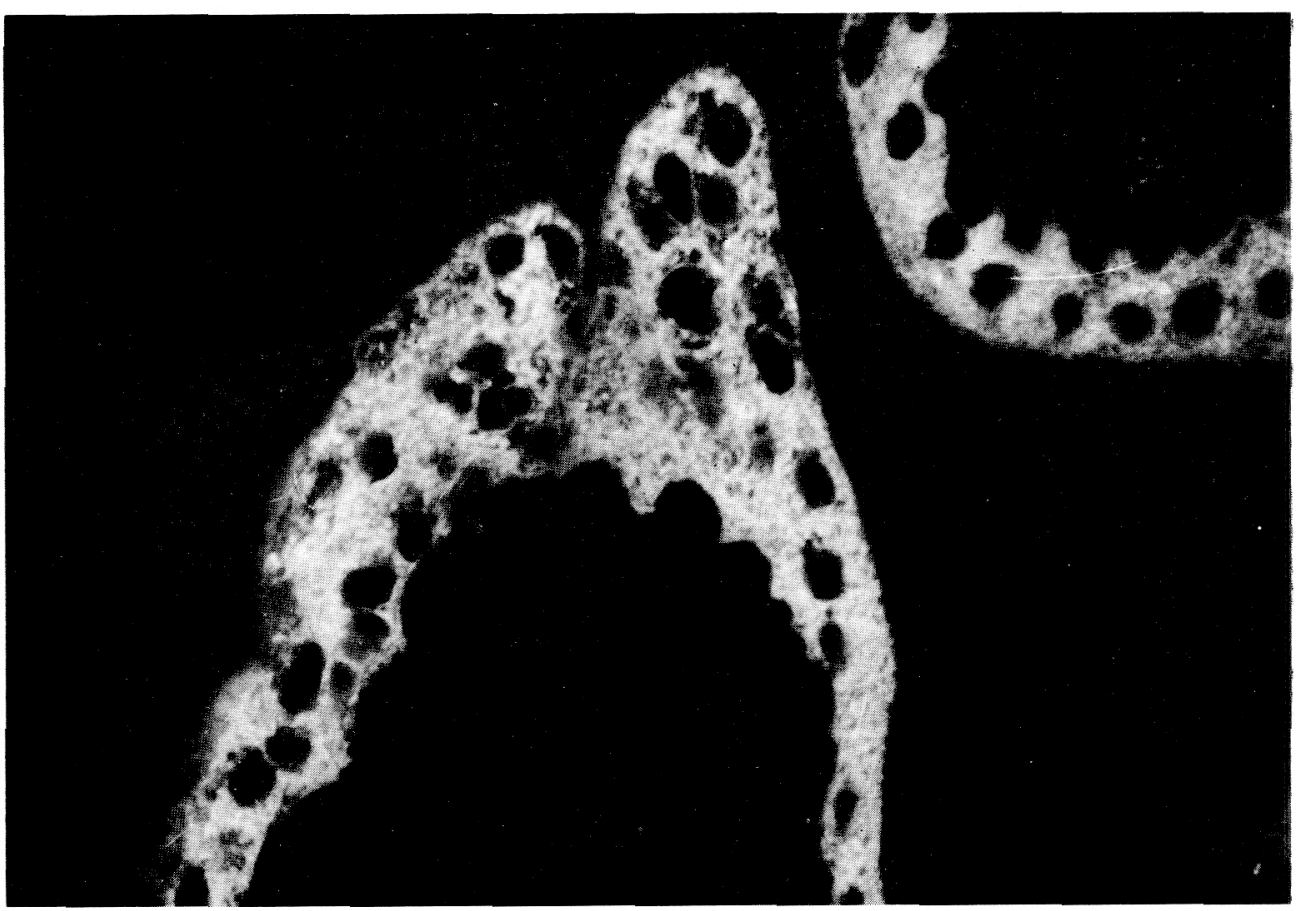

Fig. 9. Staining with anti-hCG- $\beta, \times 400$ 


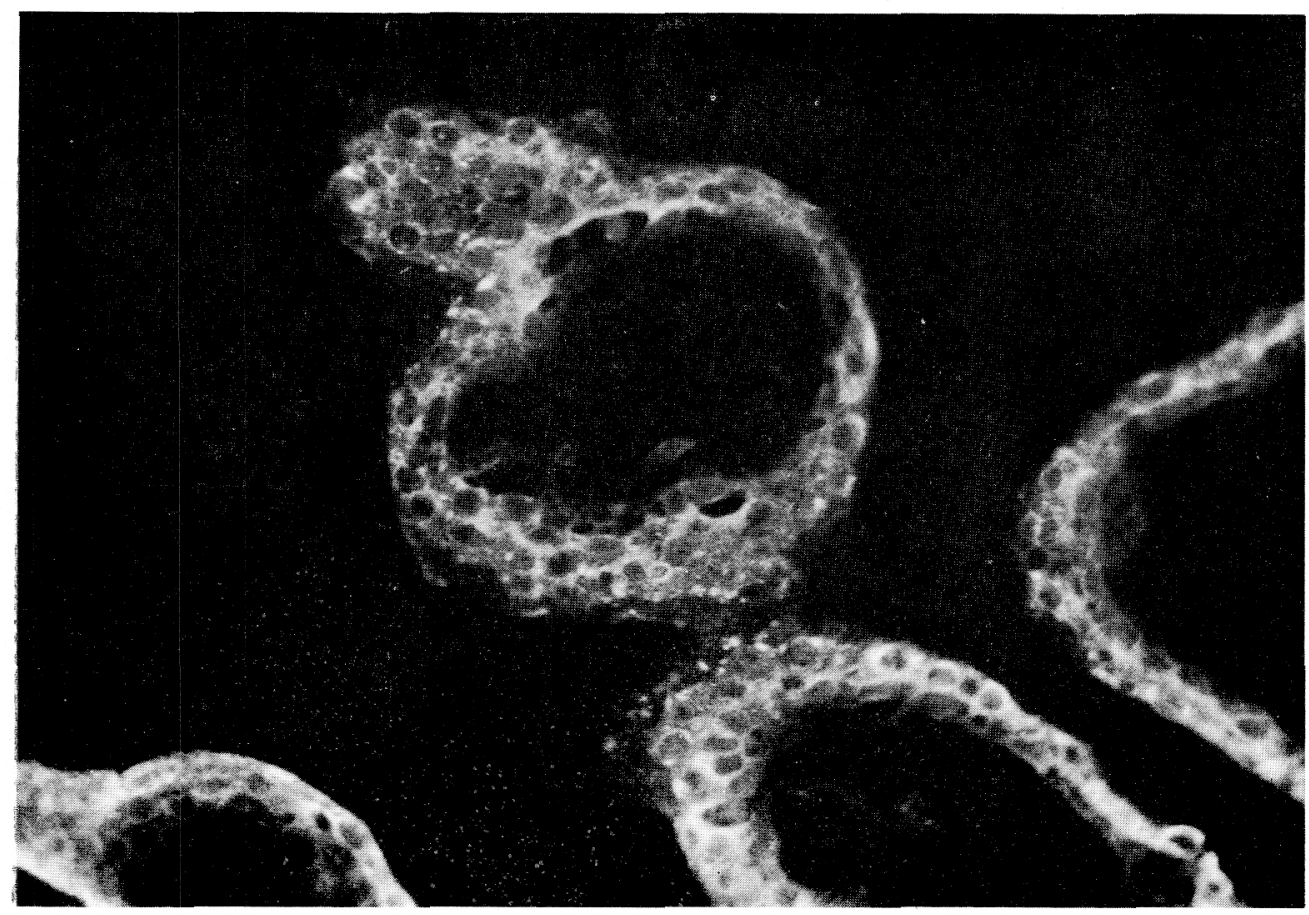

Fig. 10. Staining with anti-hCG- $\alpha, \times 160$

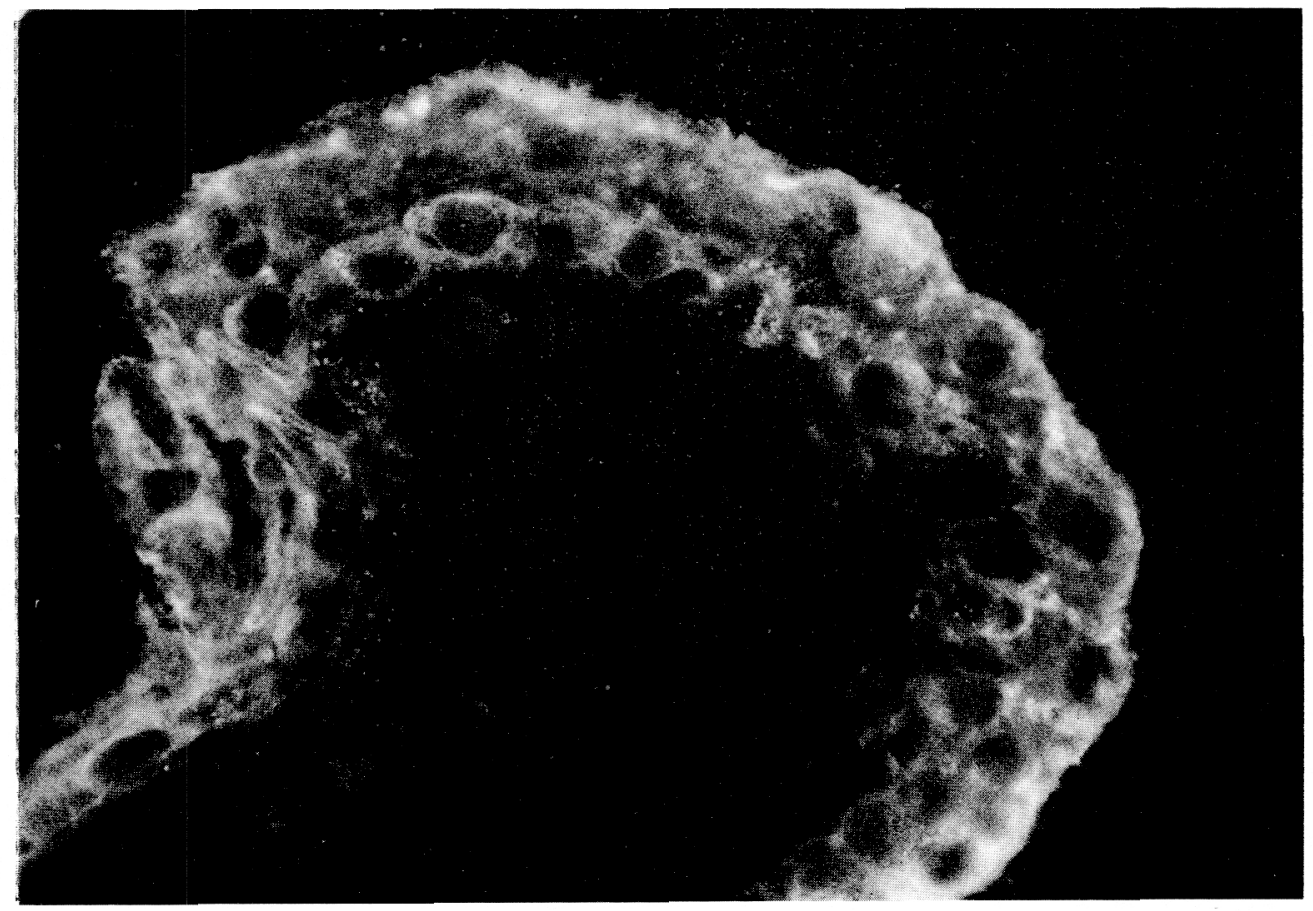

Fig. 11. Staining with anti-hCG- $\alpha, \times 400$ 


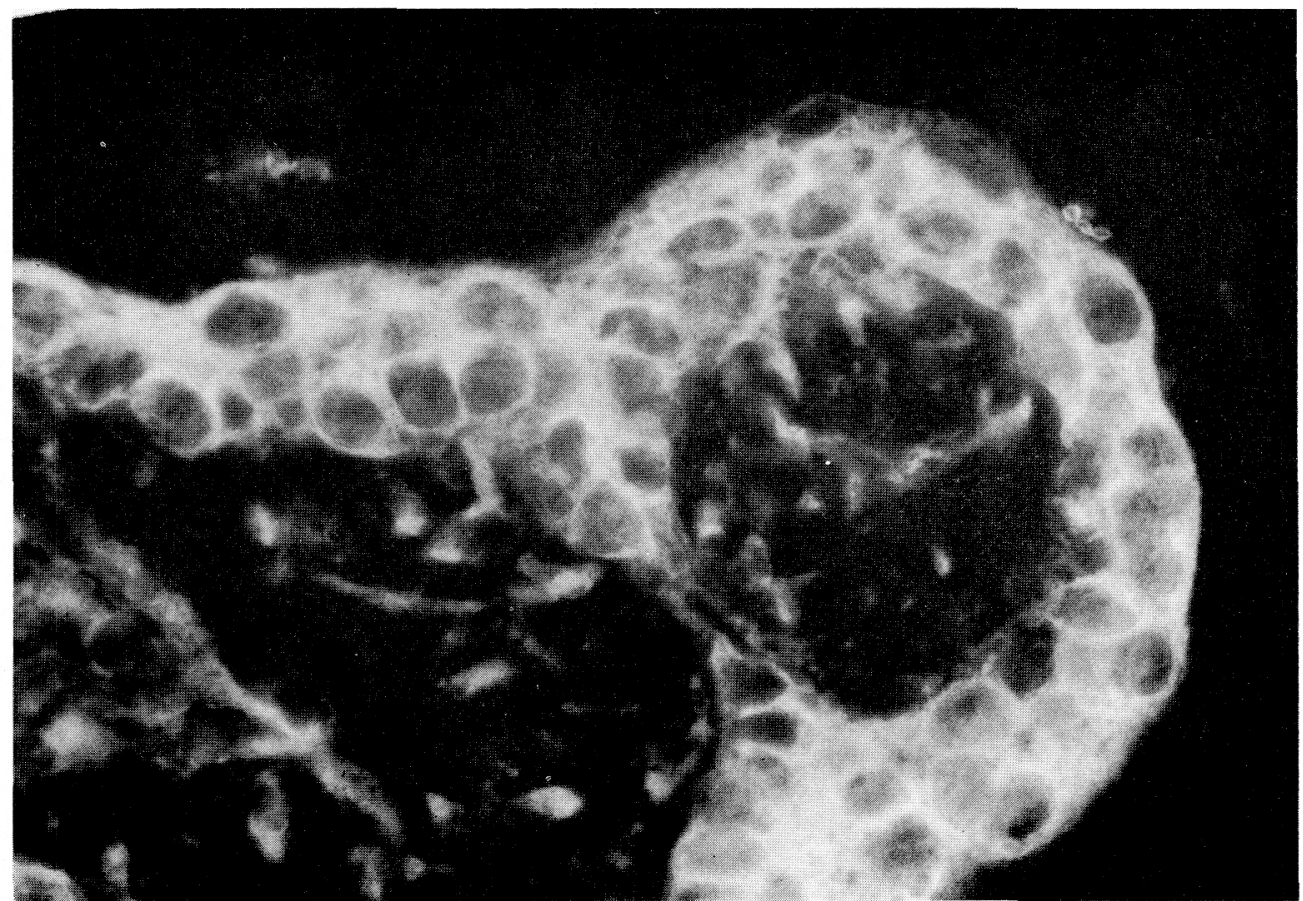

Fig. 12. Staining with FITC-conjugated anti-hCG- $\alpha$ after treatment of anti-hCG, $\times 400$

In contrast to the former two stainings, the fluorescence obtained with anti hCG- $\alpha$ was found both in the syncytio- and cytotrophoblast. The intensity of fluorescence in cytotrophoblast was variable and paler than that in syncytiotrophoblast (Fig. 10, Fig. 11). The treatment of anti-hCG prior to the staining of FITC-conjugated antihCG- $\alpha$, diminished the intensity of fluorescence in syncytiotrophoblast, and then both trophoblast showed almost the same intensity of apple green color (Fig. 12).

\section{Discussion}

The fluorescence stained with rabbit anti-hCG in normal immature placenta was observed in the outer layer of chorionic tissue, the so-called syncytiotrophoblast, as reported by many workers (Midgely et al., 1962, Notake et al., 1962, Tojo et al., 1968,
Thiede et al., 1970, Tanizawa et al., 1970, Loke et al., 1972, Gartner et al., 1975). This finding demonstrates that hCG is present in the cytoplasm of syncytiotrophoblast. However, Notake (1962), Tojo (1968) and Thiede (1970) described that a number of cytotrophoblasts did show a pale fluorescence stained with anti hCG, suggesting that hCG might exist in cytotrophoblast.

Table 1 is a summary of immunofluorescent observations by the indirect method and shows that cytotorphoblasts of immature placenta have a pale apple color

Table 1. Immunofluorescence of Normal Villi with Indirect Staining

\begin{tabular}{lccc}
\hline \hline & $\begin{array}{c}\text { Syncytial- } \\
\text { trophoblast }\end{array}$ & $\begin{array}{c}\text { Cyto- } \\
\text { trophoblast }\end{array}$ & $\begin{array}{c}\text { Interstitial } \\
\text { cell }\end{array}$ \\
\hline Anti-hCG & ++ & \pm & - \\
Anti-hCG- $\beta$ & ++ & - & - \\
Anti-hCG- $\alpha$ & ++ & + & - \\
\hline
\end{tabular}


stained with anti-hCG. Nevertheless, it seems likely that anti-hCG cross reacts with free hCG- $\alpha$ in cytotrophoblast which is considered to contain hCG- $\alpha$. The antiserum used by us is highly specific showing less than $1 \%$ cross reaction with hCG- $\alpha$ that the cytotrophoblastic fluorescence stained with anti-hCG is indicative of the presence of a small but significant amount of hCG.

As a matter of fact, hCG was detected in umbilical blood and Lauritzen (1973) postulated that $\mathrm{hCG}$ in fetal circulation influenced fetal steroidogenesis. Moreover, the fluid of molar vesicle contain relatively high amounts of hCG. From these results, it is reasonable to conclude that hCG produced by the syncytiotrophoblast is transported not only into maternal circulation but also into the fetal circulation via cytotrophoblast possesses a small amount of hCG.

Incidentally, the cellular site of production of hCG has not been clarified, nevertheless it is understood that hCG is produced by, stored in and secreted from syncytiotrophoblast for the following reasons. Electromicroscopic study showed that syncytiotrophoblast is rich in organelles and has well developed endoplasmic reticulum (Boyd et al., 1954, Wislocki et al., 1955, Pierce et al., 1963, Wynn et al., 1972). Moreover, Yoshida (1964) demonstrated merocrine like secretary granules in syncytiotrophoblast of normal immature placenta and Dreskin et al. (1970) also observed anti-hCG reacting products in endoplasmic reticulum of syncytiotrophoblast by immunohistochemistry. On the contrary, based on autoradiographic observations of chorionic villi syncytiotrophoblast has minimal activities of RNA synthesis and protein synthesis (Hoshina, 1977, Maruo et al., 1974). In addition to the findings that free $\alpha$-subunit exists in cytotrophoblast, this is at variance with the concept that hCG is produced only by syncytiotrophoblast.
On the basis of time course autoradiographic observation that the protein synthesised in cytotrophoblast is transported into syncytiotrophoblast and that the translocation rate of the protein is much higher than that of transformation from cytotrophoblast to syncytiotrophoblast, it seems likely that the proteins synthesised in the cytotrophoblast are not only structural proteins but also secretory proteins. From these data, it is meaningless to speculate on the site of hCG production because the amount of $\mathrm{hCG}$ is less than $1 \%$ of the total protein in the chorionic villi. Moreover, to establish that the syncytiotrophoblast is the site of hCG production requires further studies.

The fluorescence stained with antihCG $\beta$ is restricted to syncytiotrophoblast, indicating that $\mathrm{hCG}-\beta$ is found only syncytiotrophoblast accompanied a small amount of hCG in cytotrophoblast. As indicated in Table 1, fluorescence stained with antihCG- $\alpha$ was observed both in the syncytioand cytotrophoblast. Therefore the fluorescence of latter cells should be due to free $\alpha$-subunit since there was no fluorescence stained with the another two antisera.

On the other hand, the anti-hCG- $\alpha$ fluorescence in syncytiotrophoblast originated from both hCG- $\alpha$ and cross reaction with hCG present in large amounts. Namely, the concentrations of hCG, hCG- $\beta$ and hCG- $\alpha$ in normal immature placenta reported by Ashitaka (1974) are 10,000$13,000 \mathrm{ng}, \quad 500-400 \mathrm{ng}, \quad 500-1,500 \mathrm{ng}$ per $10 \mathrm{mg}$ of dried chorionic villi respectively. Based on this findings, the hCG in specimen was masked by treatment with anti-hCG prior to the reaction of the specimen with FITC conjugated anti hCG- $\alpha$. By this sequence of treatments, the intensity of fluorescence in the syncytiotrophoblast was markedly reduced to the extent that both trophoblasts showed about the same intensity. From these results, free hCG- $\alpha$ presumably exists both in the syncytio- and 
cytotrophoblasts at about the some concentration. This finding may explain the relatively high concentration of hCG- $\alpha$ in umbilical blood and in the fluid of molar vesicles (Ashitaka, 1977).

Dhung (1969) and Franchimont (1972) showed the synthesis of hCG and its subunits in chorionic tissue and Ashitaka reported that the blood concentration of hCG- $\alpha$ which increased with the course of gestation disappeared rapidly after delivery. All these findings suggest that hCG- $\alpha$ might be synthesized by syncytio- and/or cytotrophoblast because hCG- $\alpha$ was detected in a relative high concentration in the fluid of molar vesicle and absent from interstitial cells. Based on these results, the general notion that hCG is synthesized only by syncytiotrophoblast should be reconsidered.

It is evident that cytotrophoblast transforms into syncytiotrophoblast, from the data of time course autoradiogram that ${ }^{-3} \mathrm{H}$-thymidine labelled cytotrophoblast transform into syncytium. The authors also observed that cytotrophoblast in normal immature placenta transformed into syncytium within 72 hrs. Thus, there should be so-called the intermediate type of trophoblast observed by Wynn under the electromicroscope (1972). In the present study, the authors find a variety of fluorescent intensity in the cytotrophoblast stained with anti-hCG- $\alpha$. This variation in intensity may be due to the presence of an intermediate type of trophoblast. By studying this transformation, one may be able to recognize the course of differentiation of the cytotrophoblast. On the contrary, assuming that the cytotrophoblast is the secretory cell of $h C G-\alpha$, the variation in the intensity should be due to different phases of secretion. To establish this contention further studies are necessary.

Recently, with the development of RIA, the ectopic production of hCG and its subunits in malignant neoplasia has been reported. These findings are explained on the basis that in the course of dedifferentiation, hCG producing activity is unmasked as an immature trophoblastic character and ordinarily not expressed in the course of differentiation. In other words, ectopic hCG production in malignant neoplasia may be a measureable parameter of cell differentiation. Furthermore, Vaitukaitus and his associates (1975) described that some kinds of malignant neoplasia produce, disproportionately only one subunit of hCG. Hence, to prove ectopic production by malignant neoplasia, not only hCG but also its subunits should be measured. Especially with chorionic neoplasia, the cytotrophoblastic cells which are scarcely stained poorly with anti hCG or anti hCG- $\beta$ show great mitotic activity. Therefore, staining with anti hCG- $\alpha$ as a possible index of cell differentiation may be a valuable method to ascertain the degree of malignancy.

In this paper the authors established the cellular localization of hCG and its subunits in normal immature placenta. These results raise several questions such as the participation of $\alpha$-subunit on the process of hCG synthesis or the value of hCG- $\alpha$ to recognize differentiations of cytotrophoblast and the value of hCG as a marker of differentiation of neoplastic cells.

Further studies to solve these questions will be undertaken including electromicroscopical observation.

\section{Acknowledgements}

We wish to thank Professor M. Kyogoku, Department of Pathology, Kobe University School of Medicine for his technical advices and suggestions to the present study. Dr. S. S. Koide, the polulation Council, The Rockefeller University has kindly reviewed this manuscript and given us invaluable advice. This work was partly supported by the grant, M-73-055 from The Population Council, The Rockefeller University, New York, U.S.A. 


\section{References}

Ashitaka, Y., R. Nishimura, Y. Endoh, and S. Tojo (1974a). Endocrinol. Japon., 21, 429.

Ashitaka, Yr, R. Nishimura, K. Futamura, M. Ohashi and S. Tojo (1974b). Endocrinol. Japon, 21, 547.

Ashitaka, Y., R. Nishimura, K. Futamura, and S. Tojo (1977). Endocrinol. Japon., 25, 115.

Body, J. D. and A. F. Hughes (1954). J. Anta., $88,356$.

Dhung, G., W. McLimans, J. Horszewiez and M. Hreshehyshy (1969). Am. J. Obstet. Gynec., 104, 945.

Dreskin, R. B., S. S. Spicer and W. B. Greene (1970). J. Hisoche. and Cyto., 18, 862.

Franchimont, P., U. Gaspard, A. Reuter and G. Heynen (1972). Clin. Endocrinol., 1, 315.

Gartner, A., L. I. Larsson and O. N. Sjoberg., Acta. Obstet. Gynec. Scand. 54, 161.

Hoshina, M. (1977). J. Jap. Soc. Cancer Ther., 12, 24.

Hoshina, M. Y. Ashitaka, and S. Tojo (1978). Acta. Obst. Gynaec. Jpn., 30, 187.

Lauritzen, Ch., K. Knorr (1973). Med. Klin., 68, 897.
Loke, Y., D. Wilson and R. Borland, (1972). Am. J. Obstet. Gynec. 113, 875.

Maruo, T., Y. Ashitaka, M. Mochizuki and S. Tojo (1974). Endocrinol. Japon., 21, 499.

Midgley, A. R. and G. B. Pierce (1962). J. Exp. Med., 115, 289.

Notake, Y., M. Yamaguchi, T. Nakagawa, N. Uchiyama (1965). Clin. Gynec. and Obstet. (Japan)., $19,7$.

Pierce, G. B. and A. R. Midgley (1963). Am. J. Path. 43, 153.

Tanizawa, O. (1970). Obst. and Gyne. Soc., 59.

Thiede, H. A. and J. W. Choate (1963). Obstet. Gynecol., 22, 433.

Tojo, S., K. Kasai (1968). Report on the theme comissioned by Japan. Obstet. and Gynec. Soc., 1. Vaitukaitis, J., J. B. Robbins, E. Nieschlag and G. T. Ross (1971). J. Clin. Endocr., 33, 988.

Vaitukaitis, J. L. (1975). Annals Inter Medicine., $82,71$.

Wislocki, G. B., and E. W. Dempsey (1955). Ana. Rec., 123, 133.

Wynn, R. M. and J. Davies (1964). Amer. J. Obstet. Gynec., 88, 618.

Wynn, R. M. (1972). Am. J. Obst. and Gynec., 114, 330.

Yoshida, Y. (1964). Exep. Cell Res., 34, 305. 